\title{
ERRATUM
}

François Guillot • Urs Schaltegger

Jean-Michel Bertrand · Étienne Deloule • Thierry Baudin

\section{Zircon U-Pb geochronology of Ordovician magmatism in the polycyclic Ruitor Massif (Internal W Alps)}

Published online: 20 December 2005

(C) Springer-Verlag 2005

\section{Int J Earth Sci (2002) 91(6):964-978}

In the article homepage of the online version the name of one of the authors was unfortunately given incompletely-Jean Bertrand's full name is Jean-Michel Bertrand.

The original article can be found at http://dx.doi.org/10.1007/ s00531-002-0280-0

F. Guillot $(\square)$

UMR Processus et Bilans des Domaines Sédimentaires,

Univ. Sci. Tech. Lille bâtiment SN5,

59655 Villeneuve d'Ascq Cedex, France

E-mail: Francois.Guillot@univ-lillel.fr

Tel.: + 33-320-542241

U. Schaltegger

Department of Earth Sciences, Federal Institute of Technology

ETH, 8092 Zürich, Switzerland

J.-M. Bertrand

Laboratoire de Géodynamique des Chaînes Alpines,

Université de Savoie, 73376 Le-Bourget-du-Lac Cedex,

France

Étienne Deloule

Centre de Recherches Pétrographiques et Géochimiques, BP 20, 54501 Vandoeuvre-lès-Nancy Cedex, France

T. Baudin

Bureau de Recherches Géologiques et Minières, BP 6009, 45060 Orléans Cedex, France

Present address: U. Schaltegger

Département de Minéralogie, Université de Genève,

13, rue des Maraîchers, 1205 Genève, Switzerland 